\title{
Effects of Pendant Methyl Groups and Lengths of Methylene Segments in Main-Chains on Photodegradation of Aliphatic Polyesters
}

\author{
Etsuko Fujimoto and Takashi FusImaKI* \\ Otsuma Women's University, 12 Sanbancho, Chiyoda-ku, Tokyo 102-8357, Japan \\ * DJK Techno Science Laboratories Inc., 1-20 Takada Higashi 1-chome, \\ Kohoku-ku, Yokohama 223-0065, Japan
}

(Received December 7, 1998)

\begin{abstract}
Effects of pendant methyl groups and lengths of methylene segments in main-chains on photodegradation were investigated using GPC, DMA, and FT-IR-ATR for aliphatic polyesters such as poly(ethylene succinate) (PESU), poly(1,4-butylene succinate) (PBSU), random copolymers of poly(90 mol\% 1,4-butylene-10 mol\% 1,2-propylene succinate) (PB-PPSU $)$ and poly $(80 \mathrm{~mol} \%$ 1,4-butylene-20mol\% 1,2-propylene succinate) (PB-PPSU2). These polymers degrade with UV irradiation and the degree of degradation is in the order of PB-PPSU2 $>$ PB-PPSU1 $>$ PBSU $>$ PESU. It is obvious that lengths of methylene segments in main-chains affect considerably the photodegradation and that pendant methyl groups promote photodegradation. The photodegradation of these aliphatic polyesters occurs mainly by the mechanism of Norrish II type, which causes cleavage of main chains to produce vinyl and carboxyl end groups and a resulting reduction in molecular weight. In the cases of PB-PPSU1 and PB-PPSU2, additional photodegradation probably plays an important role. This process may be photooxidation. The primary reaction causes the formation of hydroperoxides by abstraction of reactive hydrogen atoms attached to tertiary carbon atoms, combining with oxygen, and then photolysis or thermolysis of hydroperoxides give alkoxy radicals, which lead to chain scission, as well as crosslinking.
\end{abstract}

KEY WORDS Photodegradation / Aliphatic Polyesters / Poly(ethylene succinate) / Poly(1,4-butylene succinate) / Random Copolymer of Poly(1,4-butylene-1,2-propylene succinate) / Pendant Methyl Group / Gel Permeation Chromatography / Dynamic Mechanical Analysis / Fourier Transform InfraredAttenuated Total Reflection

Aliphatic polyesters having high molecular weights $\left(M_{w}: 40000-1000000, M_{n}: 20000-300000\right)$ are produced through polycondensation of glycols with aliphatic dicarboxylic acids and coupling reactions., ${ }^{1,2}$ Aliphatic polymers are designed to impart not only excellent processibility that polyethylene and polypropylene offer but also excellent physical properties similar to those of polyethylene. ${ }^{3}$ These polyesters are stable in the atmosphere but biodegradable in compost, in moist soil, in water with activated sludge and seawater, where many microorganisms are present. ${ }^{4}$ They can be incinerated with the heat of combustion relatively lower than conventional polyolefines. They would be very useful new thermoplastics to eliminate environmental problems caused by conventional plastics wastes. ${ }^{5}$

In previous papers, ${ }^{6,7}$ it was reported that the photodegradation of poly(1,4-butylene succinate) (PBSU) occurs mainly by the mechanism of Norrish II type, which causes cleavage of main chains to produce vinyl and carboxyl end groups and reduction in molecular weight.
In this work, the effects of pendant methyl groups and lengths of methylene segments in main-chains on photodegradation were studied for aliphatic polyesters, produced through polycondensation of different glycols with succinic acid.

\section{EXPERIMENTAL}

Pressed films of aliphatic polyesters used in this work, as shown in Table I, were poly(ethylene succinate) (PESU), PBSU, random copolymers of poly $(90 \mathrm{~mol} \%$ 1,4-butylene-10 mol\% 1,2-propylene succinate) (PBPPSU1) and poly $(80 \mathrm{~mol} \%$ 1,4-butylene- $20 \mathrm{~mol} \%$ 1,2propylene succinate) (PB-PPSU2), prepared according to the methods of Showa Highpolymer Co., Ltd. ${ }^{1,2}$

These films were washed with acetone for half an hour and dried in a vacuum desiccator for $24 \mathrm{~h}$.

These specimen films (thickness shown in Table I) were irradiated at about $25^{\circ} \mathrm{C}$ with a ultraviolet (UV) light of $312 \mathrm{~nm}$ wavelength, with intensity of $9 \mathrm{~mW} \mathrm{~cm}^{-2}$ (corresponding roughly to 15 times autumnal equinox

Table I. Aliphatic polyesters of succinic acid in this work

\begin{tabular}{|c|c|c|c|c|c|c|}
\hline \multirow{2}{*}{$\begin{array}{l}\text { Aliphatic } \\
\text { polyesters }\end{array}$} & \multirow{2}{*}{ Glycols } & \multirow{2}{*}{$\begin{array}{l}\left(M_{n}\right)_{0} \\
\times 10^{-3}\end{array}$} & \multirow{2}{*}{$\begin{array}{l}\left(M_{w}\right)_{0} \\
\times 10^{-4}\end{array}$} & \multirow{2}{*}{$\left(M_{w}\right)_{0} /\left(M_{n}\right)_{0}$} & \multicolumn{2}{|c|}{ Thickness $/ \mu \mathrm{m}$} \\
\hline & & & & & GPC & DMA \\
\hline PESU & 1,2-Ethanediol & 45.3 & 27.2 & 6.00 & 170 & 170 \\
\hline PBSU1' ${ }^{\circ}$ & 1,4-Butanediol & 70.3 & 19.0 & 2.70 & 150 & 150 \\
\hline PBSU2 & 1,4-Butanediol & 31.4 & 12.0 & 3.82 & 350 & \\
\hline PB-PPSU1 & $\left\{\begin{array}{l}1,4-\text { Butanediol } 90 \mathrm{~mol} \% \\
1,2 \text {-Propanediol } 10 \mathrm{~mol} \%\end{array}\right.$ & 46.3 & 14.6 & 3.15 & 340 & 190 \\
\hline PB-PPSU2 & $\left\{\begin{array}{l}\text { 1,4-Butanediol } 80 \mathrm{~mol} \% \\
1,2 \text {-Propanediol } 20 \mathrm{~mol} \%\end{array}\right.$ & 41.5 & 12.9 & 3.11 & 280 & 200 \\
\hline
\end{tabular}


sunlight $(<360 \mathrm{~nm})$ at noon in Tokyo), using a UV tube of Ultra Violet Co., Ltd. in a dark room. Irradiation periods were $24,72,120,240$, and $720 \mathrm{~h}$.

Absorbance of films was measured with a HITACHI Co., Ltd. model U-1100 spectrometer.

Gel permeation chromatography (GPC) measurements were performed with a SHODEX GPC SYSTEM24 apparatus using a poly(methyl methacrylate) (Shodex STANDARD M-75) calibration (column, KF-60M $\times$ 2+KF-603; eluent, chloroform; polymer concn, $0.2 \mathrm{wt} \%$; temp, $40^{\circ} \mathrm{C}$ ). All irradiated films were soluble in chloroform.

Dynamic mechanical analysis (DMA) measurements were carried out with a Seiko Denshi Kogyo Co., Ltd. thermal analyzer model 200 at the frequencies of $0.5,1$,

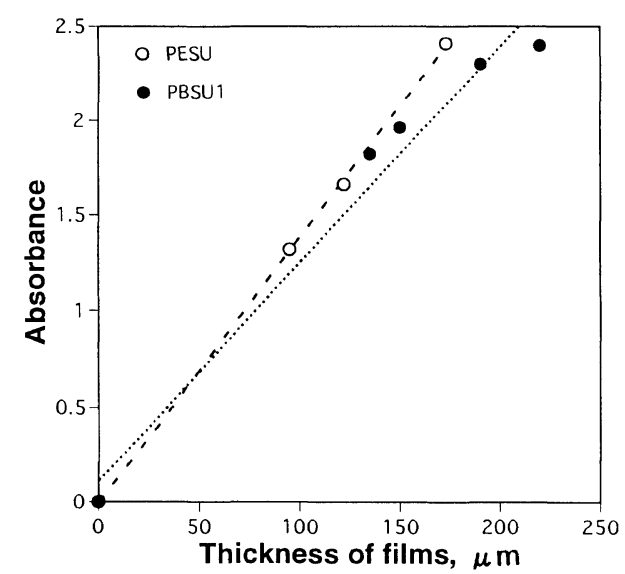

Figure 1. Absorbance at $312 \mathrm{~nm} v$ s. thickness of PESU and PBSU1 films.
$2,5,10 \mathrm{~Hz}$ and a heating rate of $1^{\circ} \mathrm{Cmin}^{-1}$ in the range of -150 to $250^{\circ} \mathrm{C}$.

Attenuated total reflection (ATR) infrared absorption spectra were obtained using a Nihon Bunko Co., Ltd. model 7300 Fourier transform infrared (FT-IR) spectrometer with 200 spectral scans averaged at $4 \mathrm{~cm}^{-1}$ resolution, fitted with a model ATR-500/M attachment with KRS-5 reflection elements.

\section{RESULTS AND DISCUSSION}

Irradiation with UV of $312 \mathrm{~nm}$ causes $n-\pi^{*}$ transition of carbonyl groups of polyesters, because these aliphatic polyesters in chloroform solutions have an absorption band at $243 \mathrm{~nm}$ tailing toward the longer wavelength. ${ }^{6}$

Figure 1 shows absorbance at $312 \mathrm{~nm}$ for thickness of films for PESU and PBSU1. It can be seen that absorbances of PESU and PBSU1 films are approximately the same.

\section{GPC Measurements}

GPC measurements were made for PESU, PBSU, PB-PPSU1, and PB-PPSU2 films irradiated with UV for various periods. The results of GPC measurements are listed in Table II, where $M_{n}$ and $M_{w}$ are number average molecular weight and weight average molecular weight, respectively. Figure 2 shows plots of $1 / M_{n}$ and $1 / M_{w}$ against irradiation time. Efficiency of chain-scission and crosslinking was determined from the slopes of the lines for cases irradiated for less than $72 \mathrm{~h}$, by the following equations $^{8}$

$$
1 / M_{n}=1 / M_{n 0}+\left(r_{\mathrm{s}}-r_{\mathrm{x}}\right) /(m) D
$$

Table II. Changes in molecular weight from GPC of polyester films irradiated for various periods

\begin{tabular}{|c|c|c|c|c|c|c|}
\hline Polyester & $\begin{array}{l}\text { UV irrad. } \\
\text { time } / \mathrm{h}\end{array}$ & $\begin{aligned} & M_{n} \\
\times & 10^{-3}\end{aligned}$ & $M_{n} /\left(M_{n}\right)_{0}$ & $\begin{array}{r}M_{w^{\prime}} \\
\times 10^{-4}\end{array}$ & $M_{w} /\left(M_{w}\right)_{0}$ & $M_{w} / M_{n}$ \\
\hline \multirow{4}{*}{ PESU } & 0 & 45.3 & 1.00 & 27.2 & 1.00 & 6.01 \\
\hline & 72 & 30.5 & 0.673 & 14.7 & 0.539 & 4.81 \\
\hline & 240 & 19.8 & 0.437 & 6.80 & 0.250 & 3.43 \\
\hline & 720 & 10.5 & 0.232 & 4.37 & 0.161 & 4.16 \\
\hline \multirow{6}{*}{$\mathrm{PBSU}^{6}$} & 0 & 70.3 & 1.00 & 19.0 & 1.00 & 2.71 \\
\hline & 12 & 50.6 & 0.720 & 15.9 & 0.833 & 3.13 \\
\hline & 24 & 27.2 & 0.387 & 9.69 & 0.509 & 3.56 \\
\hline & 72 & 21.2 & 0.302 & 7.00 & 0.368 & 3.30 \\
\hline & 120 & 18.7 & 0.266 & 6.28 & 0.330 & 3.36 \\
\hline & 720 & 6.90 & 0.098 & 2.02 & 0.106 & 2.93 \\
\hline \multirow{5}{*}{ PBSU2 } & 0 & 31.4 & 1.00 & 12.0 & 1.00 & 3.82 \\
\hline & 24 & 25.0 & 0.797 & 12.4 & 1.04 & 4.97 \\
\hline & 72 & 14.5 & 0.463 & 10.3 & 0.861 & 7.10 \\
\hline & 240 & 8.96 & 0.286 & 7.17 & 0.600 & 8.00 \\
\hline & 720 & 5.29 & 0.169 & 2.61 & 0.218 & 4.93 \\
\hline \multirow{5}{*}{ PB-PPSU1 } & 0 & 46.3 & 1.00 & 14.6 & 1.00 & 3.15 \\
\hline & 24 & 19.0 & 0.410 & 9.46 & 0.649 & 4.98 \\
\hline & 72 & 11.3 & 0.243 & 6.47 & 0.444 & 5.75 \\
\hline & 240 & 8.23 & 0.178 & 4.40 & 0.302 & 5.35 \\
\hline & 720 & 3.36 & 0.073 & 1.06 & 0.073 & 3.15 \\
\hline \multirow{5}{*}{ PB-PPSU2 } & 0 & 41.5 & 1.00 & 12.9 & 1.00 & 3.11 \\
\hline & 24 & 17.6 & 0.423 & 6.52 & 0.505 & 3.71 \\
\hline & 72 & 9.06 & 0.218 & 3.63 & 0.281 & 4.01 \\
\hline & 240 & 6.68 & 0.161 & 3.23 & 0.250 & 4.84 \\
\hline & 720 & 3.82 & 0.092 & 1.30 & 0.101 & 3.41 \\
\hline
\end{tabular}




$$
1 / M_{w}=1 / M_{w 0}+\left(1 / 2 r_{\mathrm{s}}-2 r_{\mathrm{x}}\right) /(m) D
$$

where $M_{n 0}$ and $M_{w 0}$ are initial number average molecular weight and initial weight average molecular weight, respectively, $r_{\mathrm{s}}$ and $r_{\mathrm{x}}$ are efficiency of chain-scission and crosslinking per repeating unit irradiated with unit absorbed dose, respectively, $m$ is molecular weight of a

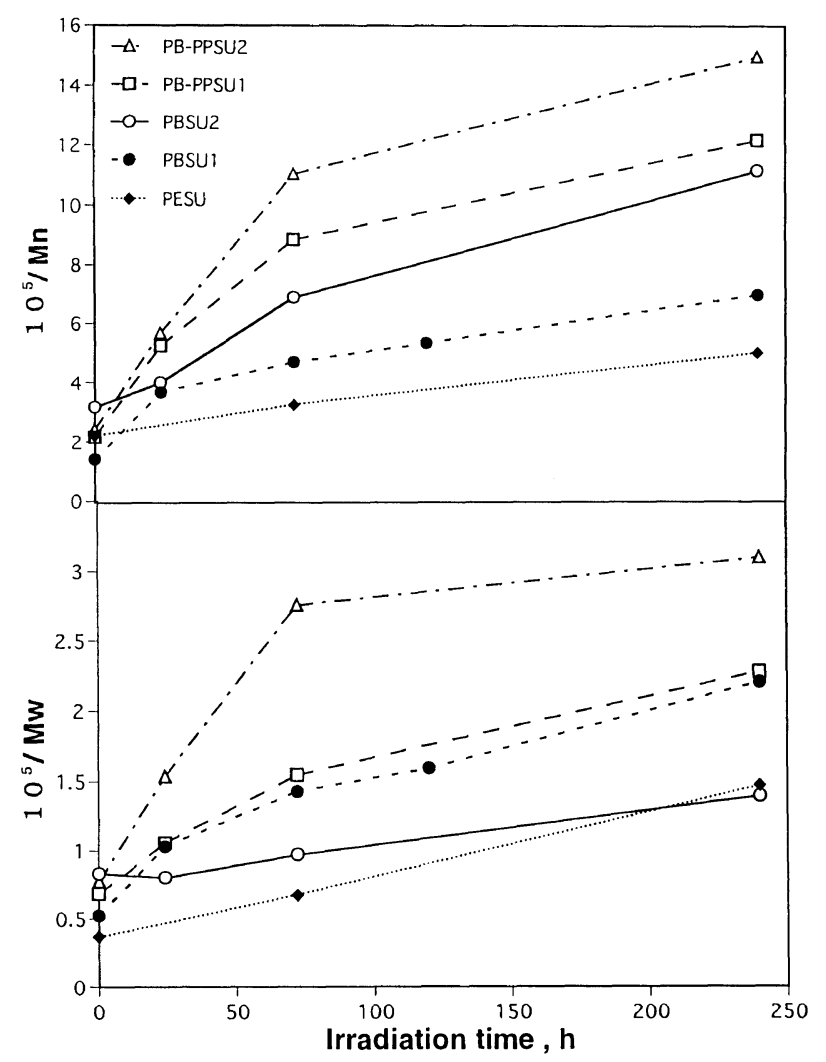

Figure 2. Change of $1 / M_{n}$ and $1 / M_{w}$ of irradiated PESU, PBSU1, PBSU2, PB-PPSU1, and PB-PPSU2 films with irradiation time. repeating unit of polymers and $D$ is the absorbed dose. The results obtained are shown in Table III. Differences between PBSU1 and PBSU2 may be due to thickness of films, because UV light does not penetrate through entire thickness, as seen in Figure 1. As seen in the table, these polymers decompose by UV irradiation, causing mainly cleavage of main-chains and the degree of decomposition is in the order of PB-PPSU2 $>$ PB-PPSU1 $>$ PBSU1 $>$ PBSU2 $>$ PESU. It is obvious from the comparison between PESU and PBSU1, with thickness approximately the same, that lengths of methylene segments in the main-chains considerably affect photodegradation. It is apparent from the comparison of PBSU2, PB-PPSU1, and PB-PPSU2, with thickness approximately the same, that pendant methyl groups promote photodegradation.

\section{DMA Measurements}

DMA measurements were made for PESU, PBSU1, PBSU2, PB-PPSU1, and PB-PPSU2 films irradiated with UV for various periods. PB-PPSU2 film irradiated for more than $120 \mathrm{~h}$ and the other films irradiated for more than $240 \mathrm{~h}$ could not be subjected to DMA analysis, because of brittleness.

In Figure 3 (a) storage modulus $\left(E^{\prime}\right)$ and (b) mechanical loss tangent $(\tan \delta)$ at the frequency of $1 \mathrm{~Hz}$ of polyester

Table III. Relative efficiency of main-chain scission and crosslinking obtained from GPC of polyester films irradiated with UV of $312 \mathrm{~nm}$

\begin{tabular}{lcc}
\hline Polyesters & $r_{\mathrm{s}} /$ Unit dose & $r_{\mathrm{x}} /$ Unit dose \\
\hline PESU & 0.10 & 0.006 \\
PBSU1 & 0.57 & 0.073 \\
PBSU2 & 0.35 & 0.079 \\
PB-PPSU1 & 0.57 & 0.11 \\
PB-PPSU2 & 0.86 & 0.15 \\
\hline
\end{tabular}

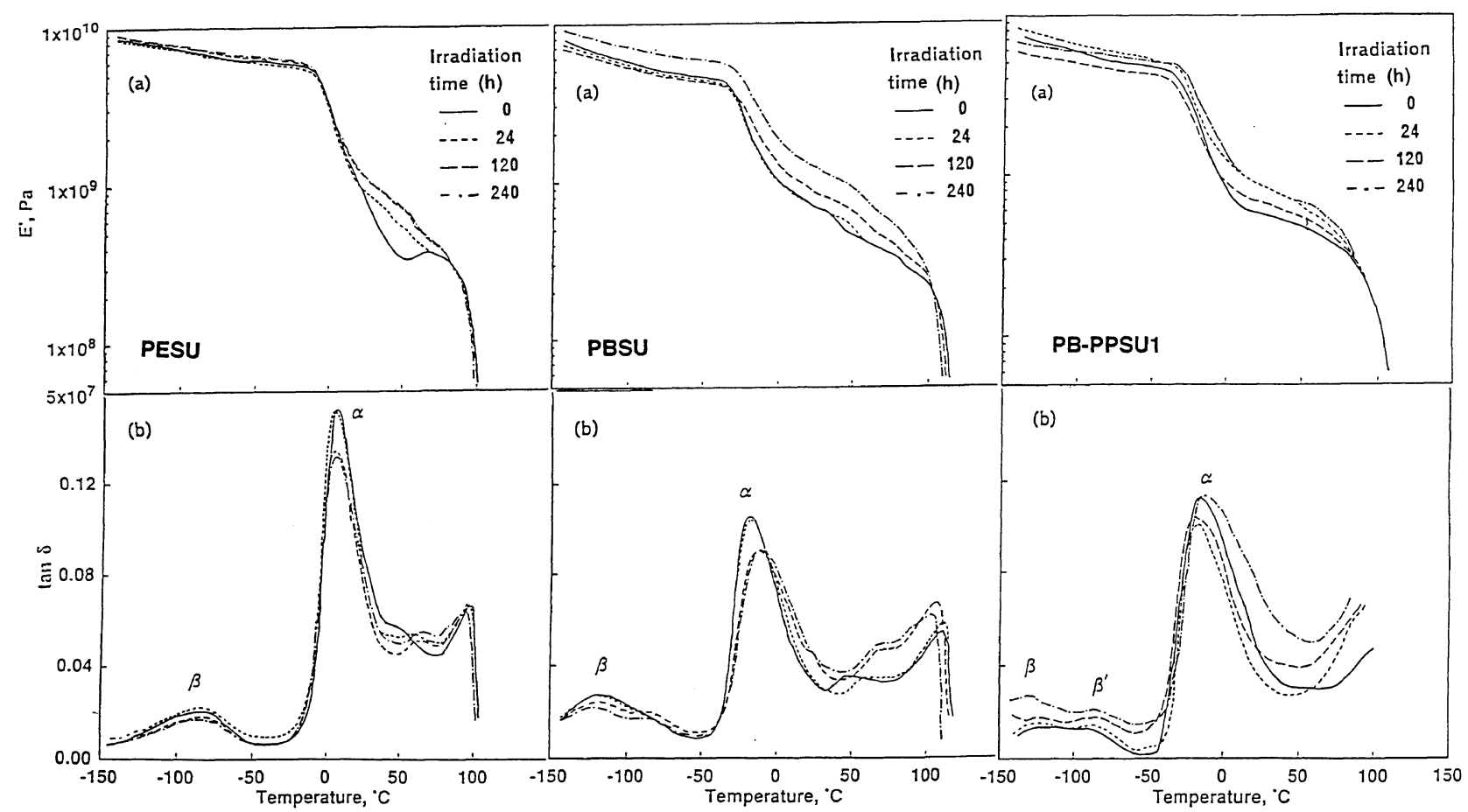

Figure 3. DMA curves of (a) storage modulus $\left(E^{\prime}\right)$ and (b) mechanical loss tangent ( $\tan \delta$ ) at $1 \mathrm{~Hz}$ for PESU, PBSU, and PB-PPSU films before and after irradiation with UV for various periods. 
Table IV. Effects of UV irradiation on $\alpha_{\max }, \beta_{\max }, \beta_{\max }^{\prime}, \Delta E_{\alpha}, \Delta E_{\beta}$, and $\Delta E_{\beta^{\prime}}$ from DMA for polyester films

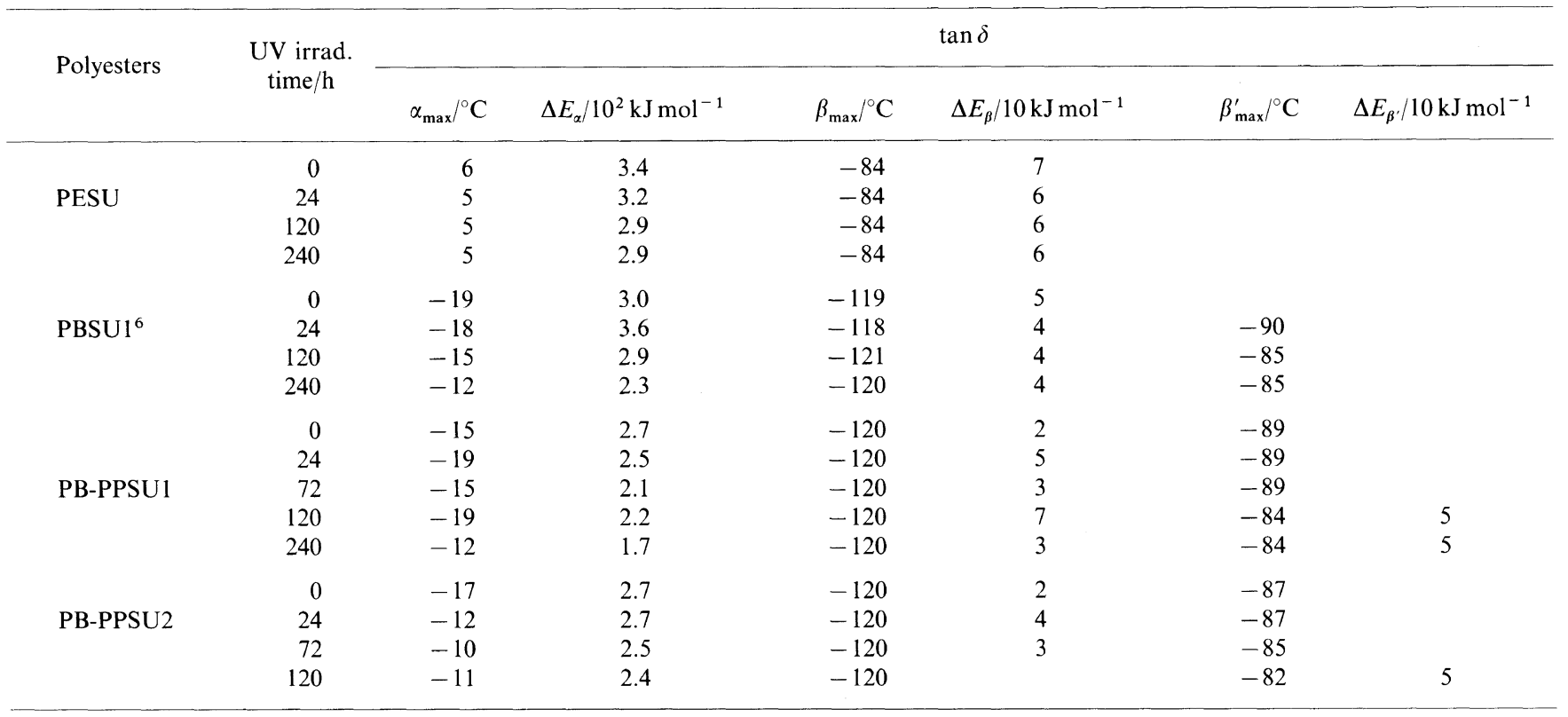

films before and after irradiation are shown as functions of temperature. In the $\tan \delta$ curve, there were primary $(\alpha)$ dispersion corresponding to micro-Brownian movement of main chains and secondary $\left(\beta\right.$ and $\left.\beta^{\prime}\right)$ dispersions corresponding to local rotational movement in sequences of methylene units (local relaxation) and intermolecular rotation of side chains, respectively. ${ }^{9,10}$ Changes in these curves during UV irradiation showed a similar tendency.

The results from dynamic experiments are listed in Table IV. In this table, activation energy $\left(\Delta E_{\alpha}, \Delta E_{\beta}\right.$, and $\left.\Delta E_{\beta^{\prime}}\right)$ was obtained from the dependence of the peak temperatures $\left(\alpha_{\max }, \beta_{\max }\right.$, and $\left.\beta_{\max }^{\prime}\right)$ on the frequency of $\alpha$, $\beta$, and $\beta^{\prime}$ dispersions, respectively.

$\Delta E_{\alpha}$ and $\Delta E_{\beta}$ before irradiation were in the order of PB-PPSU1 $\simeq$ PB-PPSU $2<$ PBSU $<$ PESU, which suggests that micro-Brownian movement in main-chains and local rotational movement may be in the order of PB-PPSU1 $\simeq$ PB-PPSU2 $>$ PBSU $>$ PESU. This order is the same as the degree of scission of main-chains obtained from GPC measurement. This may indicate that the mobility of main-chains and local rotational movements of side-chains affect photodegradation considerably.

$\Delta E_{\alpha}$ decreases after UV irradiation. This suggests that micro-Brownian movement of main-chains becomes greater during UV irradiation, indicating the disorder of the orientation of main-chains.

$\Delta E_{\beta}$ decreases in the cases of PESU and PBSU but increases in the cases of PB-PPSU1 and PB-PPSU2 during UV irradiation. The latter case indicates that local rotational movement of side-chains becomes hindered during UV irradiation, due to formation of cross-linking, as seen in Table III. This suggests that additional mechanism of photodegradation takes part in the latter.

The results from DMA measurements are consistent with those of GPC measurements.

\section{FT-IR-ATR Measurements}

The directly irradiated surfaces of the films show significant IR changes when measured by ATR spectroscopy. As shown in Figure 4, the most noticeable changes in ATR spectra are (a) the formation of broad bands centered at $3480 \mathrm{~cm}^{-1}$ attributed to hydroperoxides $^{11}$ and $3250 \mathrm{~cm}^{-1}$ attributed to $\mathrm{O}-\mathrm{H}$ in carboxyl group $^{12}$; (b) shrinking of the band at $2950 \mathrm{~cm}^{-1}$ due to $\mathrm{C}-\mathrm{H}$ and formation of unstructured shoulder in $=\mathrm{C}-\mathrm{H}$ band around $3000 \mathrm{~cm}^{-1}$, suggesting that methylene groups decrease while vinyl groups are produced during UV irradiation; and (c) the bands at $1710 \mathrm{~cm}^{-1}$ attributed to carbonyl groups shift to the lower wavenumber during UV irradiation, showing the formation of new carbonyl groups such as carboxyl group. Changes in FT-IR-ATR spectra of PB-PPSU2 on UV irradiation are approximately similar to those of PBSU ${ }^{6}$ except for the hydroxyl vibration region, as seen in Figure 4(a). During UV irradiation, a new band centered at $3250 \mathrm{~cm}^{-1}$ appears in the case of PBSU, while a new band centered at $3500 \mathrm{~cm}^{-1}$ and shoulder around $3250 \mathrm{~cm}^{-1}$ appear in the case of PB-PPSU2. This indicates the formation of hydroperoxides during UV irradiation in the latter case.

\section{Photodegradation Mechanism}

The photodegradation of PESU and PBSU is considered to occur mainly by Norrish II mechanism as shown in Figure 5 [A], ${ }^{6}$ because these polymers are able to form six-member ring structures near $25^{\circ} \mathrm{C}$, the region of transition from the glassy state to the elastic state as shown in Figure 3.

It seems that photodegradation through Norrish II mechanism occurs more easily, by increasing lengths of methylene segments in main-chains, since the flexibility of the partial chain fragment may be important in this mechanism.

For PB-PPSU1 and PB-PPSU2, partial fragments of poly(1,2-propylene succinate) (PPSU) have two formulae, namely PPSU I and PPSU II, as shown in Figure 5. It seems most reasonable that the carbon atom $(\gamma)$ of a side-chain in PPSU I takes part in forming a six-member ring structure in excited states because of the small $\Delta E_{\beta}$, similar to the case of poly(lactic acid). ${ }^{13}$

In the cases of PB-PPSU1 and PB-PPSU2, it can be 

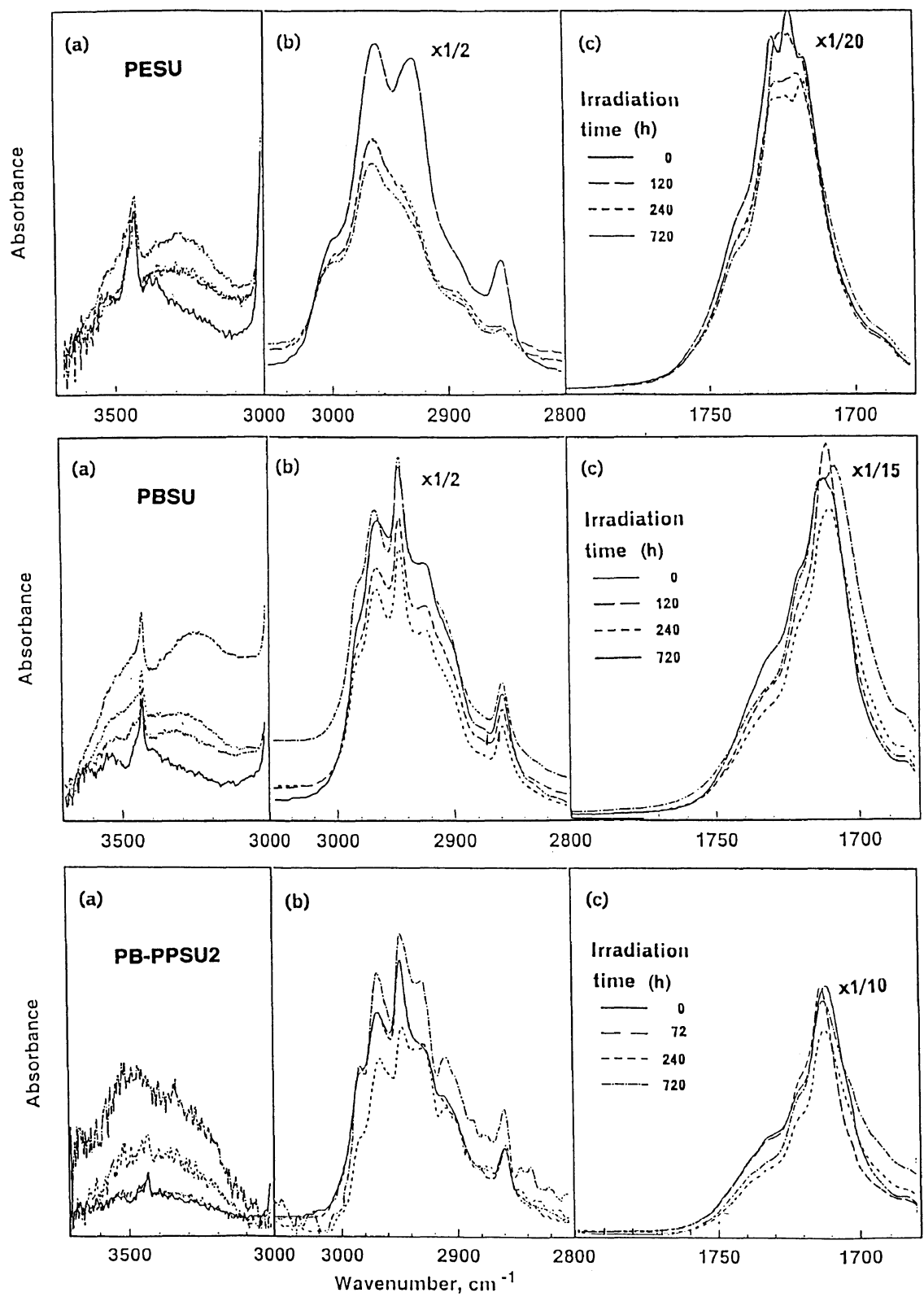

Figure 4. Change of FT-IR-ATR spectra of PESU, PBSU, and PB-PPSU2 films before and after irradiation with UV for various periods. (a) hydroxyl region; (b) $\mathrm{C}-\mathrm{H}$ region; (c) carbonyl region.

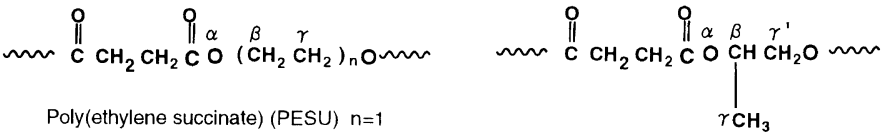

$$
\begin{aligned}
& \text { Poly(1,4-butylene succinate) (PBSU) } n=2 \quad \text { Poly(1,2-propylene succinate) (PPSU) I }
\end{aligned}
$$

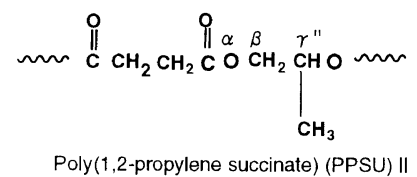

[A] Norrish II

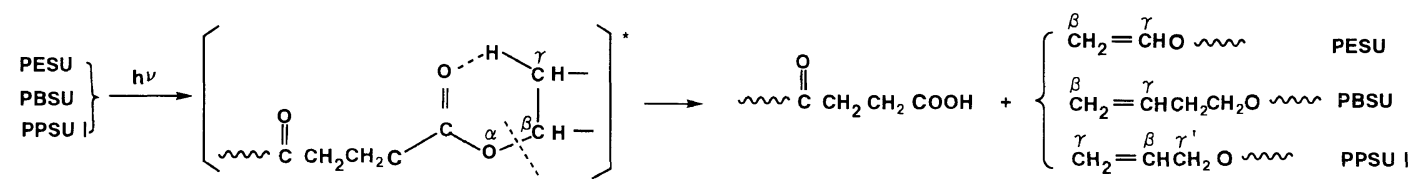

[B] Photooxidation

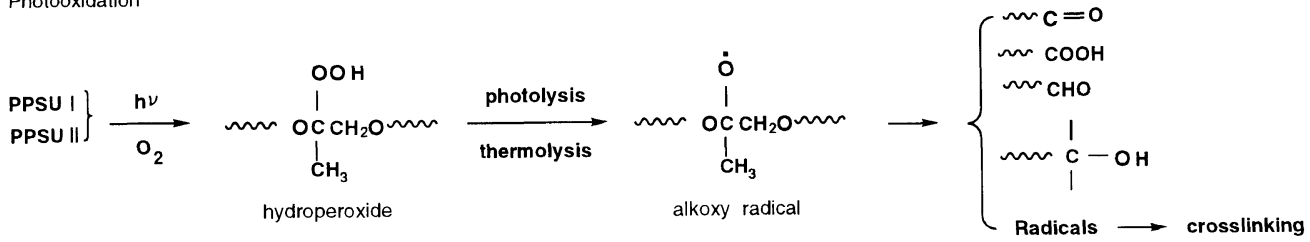

Figure 5. Photodegradation mechanism of aliphatic polyesters. 
seen from the results of DMA and FT-IR measurements that additional photodegradation probably plays an important role. This process may be photooxidation, as shown in Figure 5 [B]. The primary reaction causes the formation of hydroperoxides by abstraction of reactive hydrogen atoms attached to tertiary carbon atoms, combining with oxygen and then photolysis or thermolysis of hydroperoxides gives alkoxy radicals, which lead to chain scission, resulting in the formation of the compounds having carbonyl, carboxyl, aldehyde, and hydroxyl groups, as well as some radicals which lead to crosslinking, as reported for polypropylene. ${ }^{14}$ This postulation is supported by FT-IR-ATR study, in which a band due to hydroperoxide was observed during UV irradiation. This postulation is also consistent with experimental evidence from DMA measurements, in which $\Delta E_{\beta}$ increased during UV irradiation. Local rotational movement of pendant methyl groups is hindered, by a hydroperoxide group attached to the same carbon atom and crosslinking formed by radicals, generated in photooxidation.

Acknowledgment. The authors express sincere thanks to Shodex Service Center in Shoko Co., Ltd. for making GPC measurements.

\section{REFERENCES}

1. E. Takiyama, I. Niikura, and Y. Hatano, U.S. Patent, 5305787 (Apr. 4, 1994); M. Imaizumi, H. Kimura, R. Fujihira, Y. Ichikawa, J. Suzuki, Y. Moteki, T. Fujimaki, and E. Takiyama, Eur. Patent, 0747416 Al (1996).

2. T. Fujimaki and E. Takiyama, Polym. Prep., Jpn., 43, 3993 (1994).

3. E. Takiyama and T. Fujimaki, in "Biodegradable Plastics and Polymers," Vol. 12, Y. Doi and K. Fukuda, Ed., Elsevier, Amsterdam, 1994, p 150.

4. M. Nishioka, T. Tuzuki, Y. Wanajyo, H. Oomani, and T. Horiuchi, in "Biodegradable Plastics and Polymers," Vol. 12, Y. Doi and K. Fukuda, Ed., Elsevier, Amsterdam, 1994, p 584.

5. T. Fujimaki, Polym. Degrad. and Stability, 59, 209 (1998).

6. E. Fujimoto and T. Fujimaki, Kobunshi Ronbunshu, 52, 378 (1995).

7. E. Fujimoto, Kobunshi Ronbunshu, 55, 300 (1998)

8. Z. Osawa, "Degradation and Stabilization of Polymers," Musashino Request Co., Ltd., Tokyo, 1992, p 72.

9. H. N. Ng, A. E. Allegrezza, R. W. Seymour, and S. L. Cooper, Polymer, 14, 255 (1973).

10. G. Lunardon, Y. Sumida, and O. Vogl., Die Angew. Makromol. Chem., 87, 1 (1980).

11. N. S. Allen, M, Edge, I. R. Bellobono, and E. Selli, "Current Trends in Polymer Photo-chemistry," Ellis Horwood, Chichester, 1995, Chapter 12.

12. M. Day and D. M. Wiles, J. Appl. Polym. Sci., 16, 191, 203 (1972).

13. E. Ikada, Y. Takeuchi, and M.Ashida, Kobunshi Ronbunshu, 49, 527 (1992).

14. D. J. Carlsson and D. M. Wiles, J. Macromol. Sci.-Rev. Chem., C14, 65 (1976). 